\title{
Design Hierarchical Component-Based WebGIS
}

\author{
Yingwei Luo, Xiaolin Wang, Guomin Xiong, and Zhuoqun Xu \\ Dept. of Computer Science and Technology, Peking University, Beijing, P.R.China, 100871 \\ lyw@pku.edu.cn
}

\begin{abstract}
A practical component-based WebGIS named as Geo-Union is presented. Geo-Union consists of four layers: storage layer, service layer, component layer and application layer. Service layer can be partitioned into another two layers: client service layer and server service layer. The architectures and object constitutions of each layer in Geo-Union are discussed in details. The Web application model of Geo-Union is also presented. At last, some future works in WebGIS, such as interoperability, security, distributed computing and intelligent computing, are indicated and simply explored.
\end{abstract}

\section{Introduction}

Geographical Information System, GIS, is an effective tool that digitally reflects the geometry spatial situation on which human society lives and the various transitional spatial data. GIS describes the attributes of these spatial data and simulates the action of geospatial objects in a model way. Under the supports of software and hardware, GIS uses the given formats to support input/output, memory and display. It also provides the service of inquiring geometry spatial information, doing compositive analyses and making assistant decision. After several years' development, GIS is being wildly used in every aspect and plays an important role.

WebGIS is the Internet GIS that has Browse/Server architecture. Recently, WebGIS application has become more and more popular in many GIS user communities ${ }^{[1]}$ because of the fascinating development of computer networks as well as the more and more popular use of the Internet. The key steps to promote WebGIS to a more practical situation are rational adjusting computation functions and enhancing performance. In this paper, a practical multi-layer component-based WebGIS model Geo-Union and its Web application model are discussed.

\section{Multi-layer Component-Based WebGIS Model Geo-Union}

Component modeling is one of the main approaches to enhance functions of WebGIS. Geo-Union system is based on component technique and Client/Server architecture. The system includes four layers: storage layer, service layer, component layer and application layer, with client and server existing in service layer ${ }^{[2-4]}$, shown in Figure 1. The multi-layer component-based model enables GIS functions to be distributed in network effectively and brings high reusability of the system. Furthermore, it provides effective functions for further development and integration with other systems. 


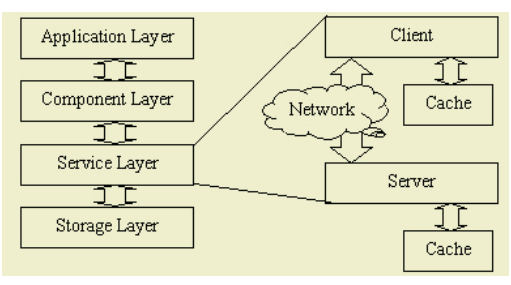

Fig. 1. Architecture of Geo-Union

\subsection{Geo-Union Storage Layer}

Storage Layer is the fundamental part in Geo-Union architecture, responsible for storage management of GIS data. With the help of ORDB, the layer stores and manages geography spatial and non-spatial data. Main objective of the layer includes how to present and store GIS data and how to maintain relations of these data. Below is type of GIS data: (1) Layer: Collection of spatial entities with the same type. (2) Entity: Spatial object composed of geometry and attribute data. Geometry data represents geometrical location of spatial object while attribute data describes society data. (3) Legend: A method to visualize Spatial Entity. (4) Legend library: Composed of $0 \sim n$ Legends. (5) Reference system: Reference frame and Attitude frame of the Layer. (6) Display Setting Item: Mapping relation between Spatial Entity and Legend. (7) Display Setting: Composed of Display Setting Items.

Figure 2 shows the relations among GIS data. Encapsulation and management of GIS data exist in all the layers: server, client and component layer, and the relation in each layer are the same.

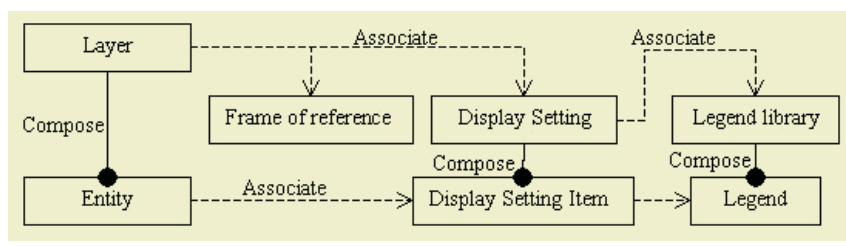

Fig. 2. E-R map in Geo-Union Storage Layer

\subsection{Geo-Union Service Layer}

Service Layer is responsible for management and access of GIS data, and composed of Geo-Union Client and Server. Geo-Union Client, as a server, provides data accessing and processing services for Geo-Union Component Layer, while Geo-Union Server manages and retrieves spatial data from Geo-Union Storage Layer, as a client. Geo-Union Client and Server are two independent but highly related parts: the Server provides functions such as data accessing service, spatial data index, basic spatial relation query, transaction and data sharing; through those services provided by the Server, the Client provides basic GIS tools and re-development functions. Cache, part 
of Geo-Union Client, can reduce network load thus improve system response rate. Cache is also in Geo-Union Server, with similar but different implementation to the Client. Geo-Union Client can be used in the development of server simulator, whose Cache enables less network load and quick system response rate.

\section{- Geo-Union Server}

Geo-Union Server is the only interface to GIS data for Geo-Union client. Through interaction with Storage Layer, the Server provides Geo-Union client with following services: connection service, data accessing service (cache service included), transaction and data sharing service. Those services are implemented mainly by a series of object component, including connection object, data object and cache object. These objects have corresponding structure and function with those in Geo-Union Client.

In Geo-Union Server, a data source table is used to manage multiple distributed spatial databases. Therefore one Geo-Union Server can serve multiple Geo-Union Clients simultaneously. Geo-Union Clients can also access multiple spatial databases through Geo-Union Server (shown in figure 3).

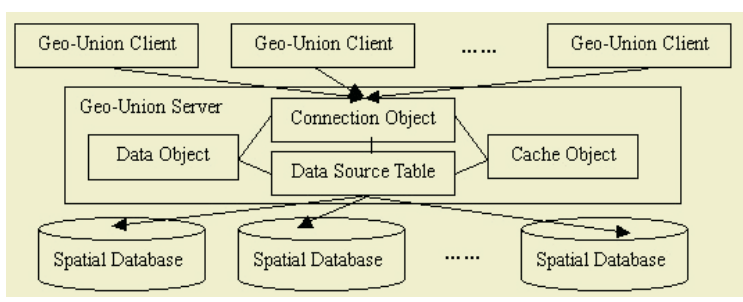

Fig. 3. Geo-Union Server and Geo-Union Client

\section{- Geo-Union Client}

Geo-Union Client is deployed on the client machine and connected with Geo-Union Server, providing GIS data accessing service and data processing service. Geo-Union client include the following functions: GIS data network access and management, GIS data object management, general spatial analysis arithmetic and spatial data cache management. Structure of Geo-Union Client is showed in figure 4. Below are the main objects in Geo-Union Client:

(1) Connection Object. Similar to Connection Object in Geo-Union Server, it manages communicating connections, GIS data access and transactions between Client and Server. It is also responsible to store and release connection relevant data objects in memory, maintain caches in Client and reduce network data flow.

(2) Data Object. Include Layer, Entity, Geometry Object, Record Collection, Map, Reference System, Display Setting, Display Setting Item, Legend Library and Legend. Considering the storage and access in Client's memory, the Client only supports two statues of Data Object: binding and dissociation. In other words, the Client can not only bind Data Object with data in Storage Layer through Geo-Union Server, but also store Data Object in its own memory. Below are status details of every kind of Data Object: (a) Layer is a collection of Entity. Layer also implements some basic spatial search operations such as K-near search, search for entities nearest to a specified entity in a layer. (b) Entity is the atomic access unit in GIS data, including Entity 
Identifier, geometry object attribute, user attribute and annotation. (c) Geometry Object includes point, multipoint, line, multi-line, polygon and bitmap. Basic spatial arithmetic of relation between Geometry Objects is also provided. (d) Record Collection. As another interface to access Entities besides Layer, Record Collection is the uniform interface to access spatial and user attributes. It can store the results of searching for entities in a layer in the form of snapshot, support cursor operation, and support both immediate and batch update modes. (e) Map manages and accesses layers' structure information in Client. Through a Map Object, the system can organize layers into a practical map.

(3) Arithmetic Object. Arithmetic Object implements general spatial analysis arithmetic such as overlay analysis, network analysis and etc.

(4) Spatial Data Cache. In Spatial Data Cache, historical records of Data Object are stored to avoid retrieving repeatedly the same data from servers, thus reducing network load and user waiting time.

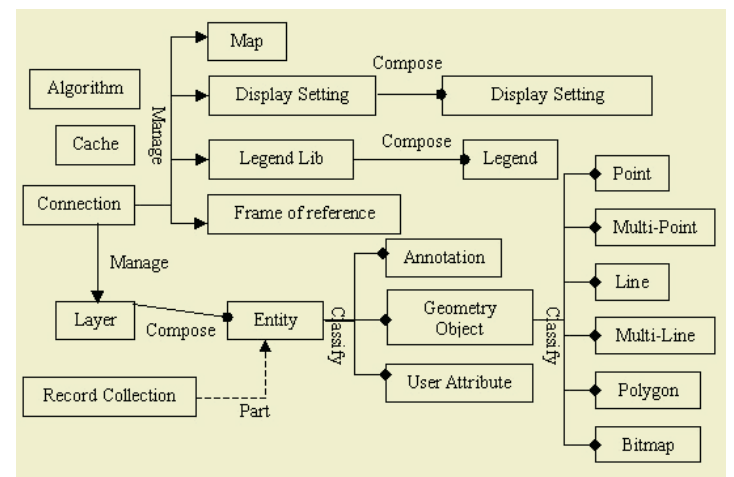

Fig. 4. Design Architecture of Geo-Union Client

\subsection{Geo-Union Component Layer}

Geo-Union Component Layer can provide Geo-Union Application Layer with many services. Geo-Union Component Layer is the encapsulation of Geo-Union Client and offers outer users GIS service interfaces to compose a complete GIS component library. The interfaces include Data Access Object, Map Display Object, Geometry Object, Function Object, Legend Edit and Display Object, Tool Object and etc. Composition and relation of these objects can be shown as figure 5 .

\section{- Data Access Object}

Data Access Object provides GIS data manipulation functions as below: (1) GxConnection, connection object for GIS data access on the server. Operations such as connection or disconnection, layer object management, reference system management, display setting object management, legend library object management and transaction are allowed in GxConnection. (2) GxLayer, layer access object. Operations such as receiving and changing layer basic information, search and analysis based on layer, managing and searching entities in a layer, importing and exporting data are allowed. (3) GxEntity, entity object, through which users can access geometry and property 
data of an entity. (4) GxLegendLib, legend library. Each legend has a number greater than 0. (5) GxLegend, used for describing a legend. (6) GxReferenceSystem, used for describing a reference system. (7) GxDisplaySetting, display setting, which describes the visualization method of a layer. (8) GxDisplaySettingItem, one item in a display setting, which describes the visualization method of a kind of entities. (9) GxRecordset, search record set, composed of searched results. (10) GxFields, field connection of an entity. (11) GxField, one field of an entity. (12) GxSelection, selected entity number connection.

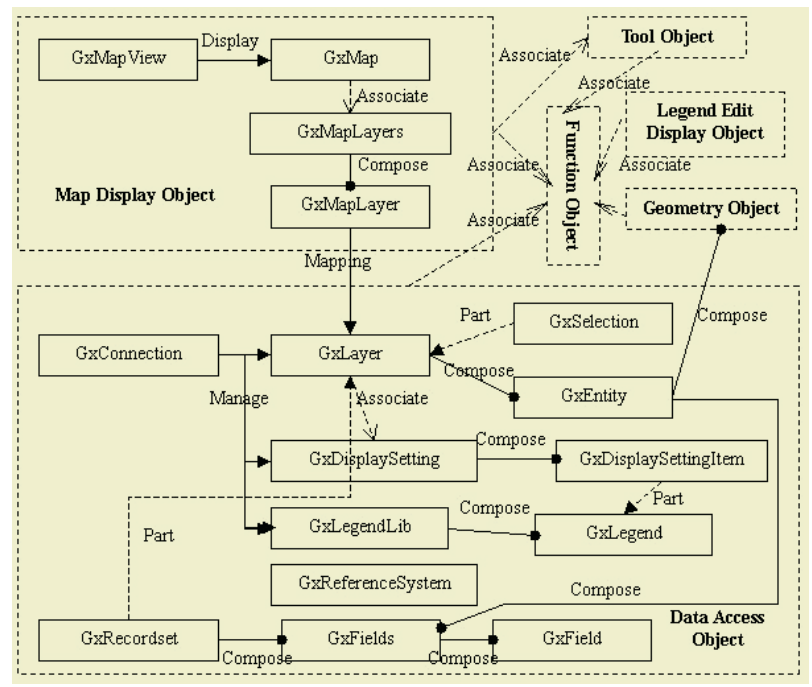

Fig. 5. Object Diagram in Component Layer of Geo-Union

\section{- Map Display Object}

Map display object can control and display GIS data, it includes: (1) GxMapView, map display control, is used for displaying map. In order to facilitate development on this control, we provide a tool management object and a group of input events. Through the tool management object GxTools, different tool objects (see also Tool Object) can be added in GxMapView to implement many special functions such as map edit, entity select, map measure and so on. The input events are all mouse or keyboard relevant and can be used in secondary development to implement customized functions. (2) GxMap is an abstract concept, can be displayed in GxMapView. One GxMap is corresponding to a collection of GxMapLayers. (3) GxMapLayers, a collection of map layers, can manage its owned map layers. (4) GxMapLayer, map layer in abstract concept, is corresponding to the map layer in storage. GxMapLayer is the map layer provided for users and includes legend configuration, reference system and annotation.

\section{- Geometry Object}

Geometry Object includes GxPoint (point entity), GxPoints (point collection), GxLine (line entity), GxLines (line collection), GxPolygon (polygon entity), GxRect (rectangle entity), GxBitmap (bitmap) and so on. 


\section{- Function Object}

Function Object has two functions: one is that it can help users utilize other objects conveniently in the secondary development process; the other function is enable users to carry on special analysis in a more convenient way. (1) GxError, error handling objec. (2) GxEnumeration, enumeration object to contain the results of enumeration methods in GxConnection such as EnumLayer (enumerate all layers), EnumLegendLib (enumerate all legend libraries), EnumReferenceSystem (enumerate all reference systems), EnumDisplaySetting (enumerate all display settings) and so on. (3) GxObjectFactory, object factory. In IIS ASP programming, only ActiveX control object s can be created directly and other programmable objects must be created through controls in a indirect way. Therefore, GxObjectFactory is designed to support the creation of GIS object in all circumstances. (4) GxArray, is a kind of array, whose element can be any type or object. (5) GxSet is a kind of set, whose element can be any type or object. No element is the set can be equal to any other element. (6) GxUtitlity, a special function utility object, contains some functions which are difficult to be implemented in some visualized programming languages such as VisualBasic, Delphi, PowerBuilder. The functions includes object creating (i.e. creating a GxPoint object by $\mathrm{x}$ and y coordinates), special statistics and coordinate transforming. (7) GxAnalysis, special analysis object, including overlay analysis, clipping analysis and connectivity analysis.

\section{- Legend Edit and Display Object}

Legend Edit and Display Object can create, edit, manage and explore legends. It includes GxLegendEditor (legend edit control), GxLengendLibView (legend library explore control) and GxLengendView (legend display control).

\section{- Tool Object}

Tool Object implements a set of basic mouse interfaces, including GxTools (tool manage object to manage a set of other tool objects), GxPick (pick entities in a map layer), GxInputLine (input line entities in a map layer), GxInputPolygon (input polygon entities in a map layer), GxInputRect (input rectangle entities in a map layer), GxZoomIn (map zoom in), GxZoomOut (map zoom out) and GxPalm (map roam).

\subsection{Geo-Union Application Layer}

In Geo-Union Application Layer, users can develop customized GIS applications. This layer's main job is to obtain customized GIS application by pruning and integrating services provided by Component Layer.

\section{Web Application Model of Geo-Union}

Geo-Union has complete functions and flexible adaptabilities. A series of techniques to construct GIS application system are provided by Geo-Union, including server construction and application development. Geo-Union not only supports stand-alone environment, but is quite suitable for developing GIS application system, especially Web-based GIS application in network environment. 
Geo-Union component layer contains a group of ActiveX controls and relative programmable objects. ActiveX controls can be embedded in Web pages directly. Web application model in Geo-Union is shown in figure 6.

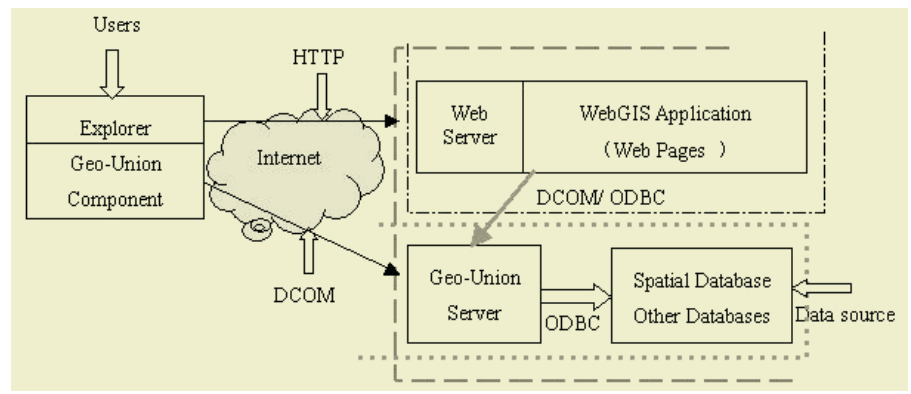

Fig. 6. Web Application Model in Geo-Union

Explorer communicates with Web server through HTTP and then gets WebGIS application module. Explorer interprets and executes the application module after receiving it. WebGIS applications are developed with Geo-Union components in specific application domain. WebGIS application and Web server compose WebGIS application server. WebGIS application can also access Geo-Union server to handle requests from Explorer.

Geo-Union server provides outer users interfaces to search and access spatial and non-spatial interfaces. As a client of Geo-Union server, Web server accesses geometry data through Geo-Union server, using ODBC or COM/DCOM. Explorer also needs accessing spatial and non-spatial data when interpreting and executing WebGIS application module. Here, as a client of Geo-Union server, explorer can search and access data using DCOM. When accessing spatial data which is transferred in vector form, explorer does not need downloading all data of the layer, but requests dynamically required entity data from Geo-Union server using entity data miss request algorithm. At the same time, as a client, Geo-Union server accesses spatial database to get spatial and non-spatial data through ODBC.

Based on commercial ORDBs, spatial database manages spatial and non-spatial data tightly relative with WebGIS application. Geo-Union server accesses database through ODBC, so the database needn't be confined in a certain specific databases.

\section{Conclusion}

Geo-Union system has been applied in many fields in China, such as pipe network integration information system, electric information system, water environment information system and fire emergency information system. At present, these systems are under stable and correct condition. What is more, the system keeps unfailing performance after repetitious visits.

Geo-Union system is also the result of research and application in some critical techniques of WebGIS. However, to reach higher practicability, many works left as 
follows: (1) As the development of Internet, more and more spatial data become available. But these data have different formats. It is still hard to share the data and services between various GIS. In order to solve this problem, people have studied standardization of GIS, but the actual open GIS has not been formed. (2) Along with the further open of Internet, the security of visiting spatial data is an unavoidable problem which WebGIS has to face. It is hoped that the secret spatial data in Web can't be achieved unlawfully. (3) WebGIS will meet the needs of thousands upon thousands users who visit Internet at the same time. How to guarantee the exactitude of concurrency and how to use the system ability farthest to meet the users' visit needs are the keys to making WebGIS worthy of its name. (4) Nowadays, intelligent agent technique is a main research direction of software domain. It provides an effective solution for establishment of complicated distributed software system. Of course, agent technique also provides a fire-new method for the establishment of WebGIS ${ }^{[6]}$. Researches on how to exert agent technique on distributed GIS construction and combine it tightly with geometry spatial metadata are not only for GIS data sharing and service sharing, but for deep GIS application cooperation and intelligent GIS information services. Furthermore, the researches provide a simple and convenient agentbased system development method for users, thus having abroad application future and important practicality ${ }^{[7]}$.

\section{Acknowledgement}

This work is supported by the National Research Foundation for the Doctoral Program of Higher Education of China under Grant No. 20020001015; the 973 Program of China under Grant No.2002CB312000; the National Science Foundation of China under Grant No.60203002; the 863 Program under Grant No. 2002AA135330 and No. 2002AA134030; the Beijing Science Foundation under Grant No.4012007.

\section{References}

1. Zhang, Li, et al: Geographic Information System in the Internet Age (in Chinese), ACTA GEODAETICA et CARTOGRAPHICA SINICA, 27(1): 9-15 (1998).

2. Luo, Yingwei, et al: The Components Design for WebGIS (in Chinese), Chinese Journal of Image and Graphics, 4(A): 79-84 (1999).

3. Li, Muhua: Research and Implementation of the Componentization of Distributed WebGIS (in Chinese), [Master Dissertation]. Beijing: Peking University (2000).

4. Wu, Jian: A Study on Spatial Data Management in Component-based Distributed WebGIS (in Chinese), [Master Dissertation], Beijing: Peking University (2000).

5. Cong, Shengri: Key Issues on ORDB-Based Component GIS (in Chinese), [PhD Dissertation], Beijing: Peking University (1999).

6. M. Wooldridge and N. R. Jennings: Intelligent Agents: Theory and Practice, Knowledge Engineering Review, 10(2): 115-152 (1994).

7. Luo, Yingwei, et al: The Research on Geo-Agents (in Chinese), Journal of Computer Research and Development, 37(12): 1504-1512 (2000). 